\title{
La ética del cuidado, sustento de la bioética enfermera*
}

\section{The ethics of care, support of nursing bioethics}

\section{A ética do cuidado, o apoio da bioética de enfermagem}

Fecha de recepción: 10 de junio de 2015

Fecha de evaluación: 7 de agosto de 2015

Fecha de aceptación: 26 de octubre de 2015

Disponible en línea: 15 de diciembre de 2015

Loreto María García Moyano**

Begoña Pellicer García***

Oihana Arrazola Alberdi***

DOI: http://dx.doi.org/10.18359/rlbi.1442

Cómo citar:

García Moyano, L. M., Pellicer García, B. y Arrazola Alberdi, O. (2016). La ética del cuidado, sustento de la bioética enfermera. Revista Latinoamericana de Bioética, 16(1), 72-79. DOI: http://dx.doi.org/10.18359/rlbi.1442

Artículo de reflexión.

** Diplomada Universitaria en Enfermería; máster en Ciencias de la Enfermería, de la Universidad de Zaragoza, España; máster en Bioética, de la Universidad Rey Juan Carlos I, Madrid, España. Enfermera del Equipo Soporte Atención Domiciliaria, Sector II, Zaragoza; miembro del Grupo de Investigación en Bioética del Instituto Aragonés de Ciencias de la Salud. Correo electrónico: lmgarciamoy@salud.aragon.es

*** Graduada en Enfermería; máster en Investigación en Ciencias de la Salud, de la Universidad San Jorge, Zaragoza, España; Becaria Pre-Doctoral-FPI. Correo electrónico: bpellicer@usj.es

**** Diplomada Universitaria en Enfermería, máster en Ciencias de la Enfermería, de la Universidad de Zaragoza, España; máster en Salud Pública, de la misma universidad. Enfermera Servicio Cardiología, Neumología y Neurología, Hospital San Jorge de Huesca. Correo electrónico: oarrazola@salud.aragon.es 


\title{
Resumen
}

En la actualidad, la enfermería ha sabido reconocer la importancia de las exigencias éticas que vinculan al acto del cuidado, y así ha dado razón a los diferentes conflictos de valores a los que los profesionales hacen frente a diario. No obstante, la profesión, no ha sabido encontrar un modelo bioético único que englobe todos los aspectos que surgen de su quehacer profesional y que ayudaría a aumentar la calidad de los servicios de salud percibida, para humanizar así la acción profesional del cuidado. El presente trabajo pretende, mediante la discusión teórica y el método de comparación constante, demostrar la necesidad de la profesión enfermera de conocer los distintos modelos bioéticos, y en especial la llamada "ética del cuidado", modelo impulsado en 1982 por la psicóloga y filósofa norteamericana Carol Gilligan, que podría servir como sustento para definir un modelo bioético enfermero concreto. De la misma manera, se pretende explicar la diferencia entre la ética del cuidado y la ética de los cuidados.

Palabras clave: ética en enfermería, ética, bioética, enfermería.

\begin{abstract}
Nowadays, nursing has been able to recognize the importance of ethical requirements linked to the act of caring, recognizing the different conflicts of value that nursing professionals must face daily. Nevertheless, the nursing profession has not yet been able to identify a single bioethical model that encompasses all the aspects that stem from its professional activity, and which would help to improve the perceived quality of health services, thus humanizing the professional act of caring. This study aims, through theoretical discussion and the constant comparison method, to demonstrate the nursing profession's need to know the various bioethical models; especially the so-called "ethics of care", a model proposed in 1982 by the American psychologist and philosopher Carol Gilligan, which may serve as the foundation to define a specific nursing bioethical model. Likewise, this article aims to explain the difference between ethics of care and ethics of caring.
\end{abstract}

Keywords: Ethics, nursing ethics, bioethics, nursing.

\section{Resumo}

Na atualidade, a enfermagem tem sabido reconhecer a importância das exigências éticas que ligam $\mathrm{o}$ ato do cuidado e, portanto, tem dado a razão a os vários conflitos de valores aos que os profissionais têm que enfrentar diariamente. No entanto, a profissão não foi capaz de encontrar um modelo único de bioética que englobe todos os aspectos que surgem do seu quefazer profissional e ajudar a aumentar a qualidade dos serviços de saúde percebidos, para humanizar assim a ação profissional do atendimento do cuidado. O presente trabalho tem como objetivo, através da discussão teórica e o método de comparação constante, demonstrar a necessidade de a profissão de enfermagem para conhecer os vários modelos de bioética, e especialmente a chamada "ética do cuidado", modelo promovido em 1982 pela psicóloga e filósofa norte-americana Carol Gilligan, que poderia servir de base para a definição de um determinado modelo concreto de bioética em enfermagem. Da mesma forma, pretende-se explicar a diferença entre a ética do cuidado e a ética dos cuidados.

Palavras-chave: ética de enfermagem, ética, bioética, enfermagem. 


\section{Introducción}

En 1977 con la incorporación de los estudios de enfermería a la Universidad, se produjo un cambio de dirección en los estudios: este hecho dotó a la enfermería de un campo específico de acción y cualificó a los profesionales en competencias independientes (Santacruz Caicedo, 2006; Alberdi Castell y Cuxart Ainaud, 2005). Incluso con esto el cuidado y su definición es lo que ha dificultado y sigue dificultando la plena profesionalización. La razón fundamental de este obstáculo es la propia naturaleza indeterminada e intangible del cuidado (Alberdi Castell y Cuxart Ainaud, 2005).

No obstante, la enfermería ha sabido reconocer la importancia de las exigencias éticas que vinculan al cuidado, es decir, a su quehacer profesional (Barrio Cantalejo et al., 2006) (Vázquez Calatayud 2011).

Una vez en ejercicio, los profesionales se hacen responsables de los actos y técnicas de su profesión. Esta responsabilidad es de dos tipos: por un lado existe la responsabilidad jurídica, y por el otro la llamada responsabilidad ética. El profesional, como cualquier otra persona es un ser moral que inconsciente o conscientemente está en una reflexión ética en cuanto a las acciones sobre la vida humana, en cualquiera de las etapas del ciclo vital. Es por ello que existe el deber de formarse en el conocimiento de los diferentes modelos de práctica ética (Alvarado García, 2004). Solo así los profesionales podrán asumir su propia percepción sobre el tema, acorde a su persona y su profesión, comprendiendo y respetando el de sus pacientes (Busquets Surribas, 2004).

El contrapunto de esta situación surge con el desarrollo de las nuevas tecnologías en la asistencia sanitaria, los elevados costes sanitarios, el aumento de las enfermedades crónicas, el avance en la genética, las discusiones sobre las acciones para llevar a cabo en la etapa final de la vida de las personas, etc. Estos cambios actuales generan dificultad en la toma de decisiones; por ello es necesario dotar de conocimientos a los profesionales para que puedan extraer del conflicto, los valores en juego y decidir sobre lo que es o no adecuado para ese individuo y su entorno más próximo (Davis, 1999).

Dichos conflictos, que en general atañen a la tecnología y al humanismo, podrían llegar a considerarse como elementos contrapuestos, cuando en realidad deben ser complementarios en cuanto a que coexisten en una misma dinámica. La relevancia actual del desarrollo tecnológico en el ámbito de la salud ha podido relegar el humanismo a un segundo plano en la asistencia sanitaria (Escuredo Rodríguez, 2003). Como apunta Escuredo Rodríguez (2003).

la tecnología, cuando olvida los valores personales y sólo busca la maximización de los resultados y se convierte en un fin en sí misma, pasa a ocupar el centro del mundo asistencial, entonces tiende a desplazar a la persona como centro de 
la atención. El enfermo pierde sus rasgos personales e individuales, se prescinde de sus sentimientos y valores y se le identifica con sus rasgos externos y accidentales, es decir, nos referimos a él por su patología o tratamiento (p. 43).

Nuestros pacientes lógicamente quieren la mejor tecnología posible, pero también precisan mayor humanismo en las relaciones sanitarias. La posibilidad de un trato humanizado se desdibuja en medio de las presiones por la optimización de recursos que, paradójicamente, buscan garantizar la universalización de la atención en el contexto de la justicia social (Pardo, 2011). La deshumanización no solo supone la insuficiente atención a las necesidades transcendentales de los pacientes, sino que también supone la falta de respeto hacia los derechos y autonomía, y además la incompleta prestación de los servicios sanitarios (Escuredo Rodríguez, 2003).

Los profesionales enfermeros no son los únicos responsables de la humanización de las prestaciones sanitarias, pero debemos contar con que la mayor presencia con el paciente nos legitima para llevar a cabo esta labor (Escuredo Rodríguez, 2003; Feito Grande, 2005b). El humanismo, aunque tiene su origen vocacional, también debe ser aprendido y practicado, sobre todo en lo referente a sus límites. Esto se consigue a través de la adquisición de competencias, habilidades de comunicación, etc., así como por medio del aprendizaje de cualidades como la prudencia, el respeto, la empatía, etc. (Escuredo Rodríguez, 2003). Es en este punto donde radica la verdadera importancia de la bioética en la profesión enfermera, usada como base para la formación en actitudes, que no entraña infravalorar los conocimientos clínicos, sino completarlos en las actitudes correctas para llevarlos a cabo, y complementar así al propio profesional (Feito Grande, 2005a).

\section{Ética del cuidado}

En 1982, la psicóloga y filósofa norteamericana Carol Gilligan, impulsora de este modelo ético, publicó el libro In a different voice, que se ha traducido al castellano como La moral y la teoría. Psicología del desarrollo femenino (Gilligan, 1983).

Concretamente, fue Lawrence Kohlberg, psicólogo, quien en 1978 planteo una investigación sobre el desarrollo moral para comprobar si al igual que en el desarrollo cognitivo (Jean Piaget) existía un desarrollo moral secuencial y por etapas, que diera como resultado una cierta maduración moral (De la Torre Díaz, 2010).

De este modo, al finalizar su investigación, Carol Guilligan corroboró su hipótesis y demostró que el desarrollo moral es secuencial. Según este modelo, un desarrollo moral conlleva asumir niveles cada vez más elevados de decisiones y acciones morales y, en consecuencia, mayor exigencia de coherencia y justificación. Aunque este modelo parece ser 
el más aceptado, sobre todo en cuanto a la certeza de la hipótesis, también ha sido objeto de numerosas críticas por la metodología utilizada. Llama poderosamente la atención el uso de dilemas hipotéticos, la ausencia de influencias culturales en los participantes, así como la exclusión deliberada por parte del investigador de aspectos emocionales (De la Torre Diaz, 2010).

La ultima crítica y quizás la más reveladora es la existencia de un sesgo de género. El psicólogo centró su estudio en los razonamientos de participantes varones (Alvarado García, 2004; Barrio Cantalejo, 1999; Fascioli 2010), y esto fue lo que impulsó a Carol Gilligan, una de sus discípulas, a investigar más a fondo sobre la cuestión.

Gilligan realiza entonces el mismo tipo de investigación que su predecesor pero con diferencias fundamentales; en primer lugar, los participantes fueron niños y niñas, y además para demostrar la hipótesis de la existencia de diferencias entre ellos en la manera de resolver los problemas de índole moral incluyó situaciones que consideraba que podrían determinar diferencias más llamativas entre hombres y mujeres (De la Torre Díaz, 2010).

En dicha obra, Gilligan desafía la concepción tradicional sobre el desarrollo moral. La escritora, que no tuvo intención de reemplazar una perspectiva por otra (Fascioli, 2010), intentó dar a conocer otra visión, una alternativa o como ella misma la definió una voz diferente. Según la autora, existen dos visiones de una misma realidad y las dos podrían ser perfectamente aceptadas. Las resoluciones o puntos de vista más impersonales de los hombres quedarían justificados por una ética de derechos o justicia. En el caso de las mujeres, su percepción ampliada, donde tienen cabida las relaciones sociales, estaría respaldada por la ética del cuidado y atención (Santacruz Caicedo, 2006).

Según dicho modelo para alcanzar los niveles más altos, en lo que al desarrollo moral se refiere, es necesaria la complementariedad de estas dos visiones. De esta manera, las emociones, que habían sido infravaloradas por un exceso de racionalismo, toman su protagonismo debido, olvidando las reducciones racionales y lógicas y exigiendo prudencia, responsabilidad e individualización en cuanto a la resolución de los problemas (Escuredo Rodríguez, 2003; Feito Grande, 2005). Nos encontramos entonces con una ética donde lo moral puede entenderse como el sentido de responsabilidad por otro ser humano, pero también como la preocupación por uno mismo. La ética del cuidado entiende que una forma de aprender y de ampliar nuestra capacidad de comprensión moral es este acercamiento o apertura hacia los demás (Hernán Baeza 2001); cultivando actitudes de relación, habilidades de comunicación y resolución de los problemas, considerando la singularidad y particularidad de cada caso y cada persona, poniendo en relieve la autenticidad de la persona, sin 
olvidarnos de lo universal y lo objetivo (Feito Grande, 2006).

\section{Conclusiones}

La ética del cuidado no es exclusiva de la enfermería; de hecho, no surge por ella y además podría aplicarse en otras muchas ciencias como la educación, la filosofía, etc. No obstante, cabe reconocer que podría ser considerada como una posible corriente de pensamiento específica (Barrio Cantalejo, 1999; Prieto Parra, 2007). Además, la enfermería ha puesto de manifiesto la importancia excesiva de las tareas técnicas que han dejado de lado la verdadera misión enfermera: el cuidado integral (Feito Grande, 2005a; Feito Grande, 2006). El cuidar y la ética del cuidar dan importancia a la relación entre enfermera y paciente, potencian el contacto y lo hacen más humano (Hernán Baeza, 2001; Davis, 2006), y esto no solo se ejerce en un momento aislado ni cuando surge un conflicto, debe ser una actitud constante (Barrio Cantalejo, 1999).

Es justo destacar que, aunque la aportación de Carol Gilligan sirvió a la enfermería por su transcendencia y ha servido de inspiración y fundamentación de la ética enfermera (Feito Grande, 2006), hubo antes enfermeras que se dieron cuenta de la importancia del cuidado y de las relaciones y escribieron sobre ello. La primera en escribir sobre este tipo de relación de un modo conceptual fue Hildegard Peplau y posteriormente también debemos reconocer la aporta- ción de la teórica Madeleine Leininger, que destacó la dependencia existente entre el cuidar y el contexto, y esto no deja de ser un escollo para desarrollar una ciencia del cuidar (Davis, 2006).

Es necesario entonces saber diferenciar la ética del cuidado y la ética de los cuidados. En cuanto a la primera, no podemos "apropiarnos" de una reflexión universal sobre la necesidad de cuidado de las personas, en cuanto a que puede pertenecer o ser tomada por otras ciencias como la filosofía o la ética (Feito Grande, 2005a) (Arroyo Gordo, 2003). Por esto resulta fácil entender que la ética del cuidado se sitúa en un ámbito diferente al de la enfermería, puede y podría aplicarse con ella, pero no es definitorio de ella, puesto que se trata de un modelo ético, es decir, un posicionamiento ético que implica unos comportamientos y actitudes en concordancia con ella. Un tipo de ética basada en la solicitud que se podría enlazar con modelos de ética de la virtud y responsabilidad (Feito Grande, 2005a; Perdigao, 2011.

En cuanto a la ética de los cuidados, que podría tener como sustento la ética del cuidado, debería ser un modelo o teoría perteneciente en exclusiva a la enfermería, basado en la responsabilidad hacia el otro y, en consecuencia, en su atención integral por medio del fomento de actitudes adecuadas y que dotaría a quien lo practica de un compromiso profesional y moral. Para llevarla a cabo, para llevarla a la práctica, son fundamentales, como en cualquier otro 
modelo enfermero, unas normas que no solo se basen en los afectos por aquellos con los que compartimos nuestra existencia, sino que dichos afectos por personas que nos son menos cercanas surgieran del respeto y la comprensión (Feito Grande, 2006). Dichas normas deberían englobar también los aspectos más técnicos de la profesión, en la actualidad de innegable importancia. Una persona tiene el deber de ayudar a los demás, y es algo tan humano y constitutivo que no existe ni la duda ni la omisión (Prieto Parra, 2007), y aún más cuando esta es la base de su profesión. Pero tampoco bastaría con esto; la ética de los cuidados incluiría también el arte enfermero, el cuidado en sí, y de esta manera haría hincapié por igual en aspectos técnicos, relacionales y en los llamados cuidados invisibles.

De hecho, algunos autores se han afanado por demostrar que las enfermeras y los pacientes se sienten más realizados cuando se enlazan los dos significados del cuidar: el proporcionar cuidados y la preocupación en la práctica clínica diaria y para ello creen realmente necesario el conocimiento y la aplicación de la ética del cuidado (Alberdi Castell y Cuxart Ainaud, 2005; Davis, 2006).

\section{Agradecimientos}

Nuestro agradecimiento a Elena Postigo, María Teresa Delgado, Rogelio Altisent y África Moyano, por el esfuerzo hacia su profesión, generosidad, ayuda, apoyo y colaboración.

\section{Referencias}

Alberdi Castell, R. M. y Cuxart Ainaud, N. (2005). Cuidados, enfermeras y desarrollo profesional: una reflexión sobre las bases del ejercicio profesional. Presencia, 1(2).

Alvarado García, A. (2004). La ética del cuidado. Aquichan, 4(4), 30-39.

Arroyo Gordo, M. P. (2003). La ética del cuidado y la ética del cuidar. Metas Enfermería, 53(6).

Barrio Cantalejo, I. M. (1999). Carol Gilligan y la ética del cuidado. Un referente para la ética de enfermería. Enfermería Clínica, 9(2), 71-76.

Barrio Cantalejo, I. M., Molina, A., Sánchez, C. M. y Ayudarte, M. L. (2006). Ética de enfermería y nuevos retos. Anales del Sistema Sanitario de Navarra, 29(3), 41-47.

Bonilla Ballesteros, A. R. (2005). Análisis comparativo de cinco teorías sobre el desarrollo moral (Tesis doctoral). Bogotá, Pontificia Universidad Javeriana.

Busquets Surribas, M. (2004). La ética del cuidar. $5^{\circ}$ Congreso Virtual en Psiquiatría y Neurociencias, Intersalud.

Davis, A. J. (1999). Las dimensiones éticas del cuidar en enfermería. Enfermería Clínica, 9(1), 21-28.

Davis, A. J. (2011). El cuidar y la ética del cuidar en el siglo XXI: qué sabemos y qué debemos cuestionar. Collegi Oficial d'Infermeria de Barcelona. Recuperado de http://www.coib.org/uploadsBO/noticia/documents/ANNE\%20DAVIS\%20 CASTELL\%C3\%A0_DEF.PDF

De la Torre Díaz, J. (2010). Mujer, mujeres y bioética. Madrid, España: Universidad Pontificia Comillas, D.L. 
Escuredo Rodríguez, B. (2003). Humanismo y tecnología en los cuidados de enfermería desde la perspectiva docente. Enfermería Clínica, 13(3), 164-170.

Fascioli, A. (2010). Ética del cuidado y ética de la justicia en la teoría moral de Carol Gilligan. Actio, (12), 41-57.

Feito Grande, L. (2005a). La ética del cuidado como modelo de la ética enfermera. Metas Enfermería, 8(8), 14-18.

Feito Grande, L. (2005b). Los cuidados en la ética del siglo XXI. Enfermería Clínica, 15(3), 167-174.

Feito Grande, L. (2006). Enfoques consecuencialistas: la ética del cuidado. Material docente Máster en Bioética. Universidad de Las Palmas.

Gilligan, C. (1983). In a different voice: psychological theory and women's development. Cambridge: Harvard University Press.
Hernán Baeza, R. (2001). Valores y comunicación. Ética del cuidado. Medicina General, 31, 113-120.

Pardo, A. (2011). La ética en la medicina. Persona y Bioética, 15(2), 166-183.

Perdigao, A. C. (2011). A ética do cuidado na intervenção comunitária e social: os pressupostos filosóficos. Análes de Psicológica, 21(4), 485-497. Recuperado de:http://www.scielo. oces.mctes. pt/scielo.php?script=sci_arttext \&pi$\mathrm{d}=$ S0870-82312003000400007\&ln$\mathrm{g}=\mathrm{pt} \& \mathrm{nrm}=\mathrm{iso}$

Prieto Parra, G. I. (2007). Perspectiva bioética del cuidado de enfermería. Enfermería Universitaria, 4(3), 21-26.

Santacruz Caicedo, M. C. (2006). Ética del cuidado. Revista Facultad Ciencias de la Salud, 8(2), 45-51.

Vázquez Calatayud, M. (2011). Filosofía y ciencia de la disciplina enfermera. Metas de Enfermería, 14(8), 67-70. 